\title{
Operative Genitalangleichung bei Mann-zu-Frau- Transsexualität: Gibt es Leitlinien oder Standards?
}

\section{Genital Reassignment Surgery in Male-to-Female Transsexuals: Do we have Guidelines or Standards?}

\author{
Autoren \\ M. H. Sohn, M. Hatzinger, K. Wirsam \\ Institut \\ Urologie, Markus-Krankenhaus, Frankfurt
}

\author{
Schlüsselwörter \\ - interdisziplinäre plastische \\ Chirurgie \\ - Transsexualismus \\ urologische plastische \\ Chirurgie \\ - Genitalien \\ - freie Lappenplastiken
}

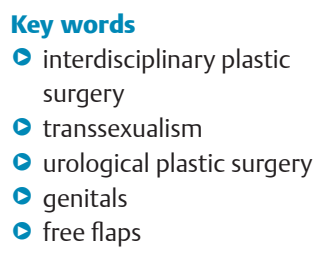

eingereicht 14.2.2013 akzeptiert 22.3.2013

Bibliografie

DOI http://dx.doi.org/ 10.1055/s-0033-1343427

Online-Publikation: 9.7.2013 Handchir Mikrochir Plast Chir 2013; 45: 207-210

(c) Georg Thieme Verlag KG Stuttgart · New York ISSN 0722-1819

\section{Korrespondenzadresse}

Prof. Dr. med. Michael

H. Sohn

Urologie

Markus-Krankenhaus Wilhelm-Epstein-Straße 4 60431 Frankfurt

Marianne.Dohman@fdk.info

\section{Zusammenfassung \\ $\nabla$}

Hintergrund: Seit 2011 liegen die „Standards of Care“ zur Diagnostik und Behandlung von Transsexuellen von der WPATH (World Professional Association for Transgender Health) in der erweiterten siebten Version vor. Nachdem auch in den letzten Jahren mehrere Übersichtsarbeiten auf dem Gebiet der genitalen Geschlechtsangleichung erschienen sind, soll untersucht werden, ob für das operative Vorgehen der genitalen Geschlechtsangleichung bei Mann-zu-Frau-Transsexuellen evidenzbasierte Leitlinien oder Standards entstanden sind.

Ergebnisse: Die Indikation zur Operation ist inzwischen weitgehend standardisiert und evidenzbasiert. Die entscheidenden Schritte der Operation sind ebenfalls mit dem Empfehlungsgrad B abgesichert. Von fast allen Autoren wird die primäre Verwendung penoskrotaler gestielter Lappen zur Auskleidung der Neovagina favorisiert. Es besteht kein Konsensus über Techniken zur idealen Rekonstruktion der Vulva, insbesondere des klitoro-labialen Komplexes. Da die Häufigkeit postoperativer Korrektureingriffe auch an sehr erfahrenen Zentren über $50 \%$ beträgt, favorisieren einigen Autoren ein primär 2-zeitig intendiertes Vorgehen.

Schlussfolgerung: Indikation und prinzipielle Technik der operativen Genitalangleichung bei Mann-zu-Frau-Transsexuellen sind evidenzbasiert abgesichert. Die subjektive Zufriedenheit der betroffenen Patientinnen liegt bei Beachtung der vorliegenden Empfehlungen über 80\%.

\section{Einleitung}

$\nabla$

1997 wurden durch alle 3 deutschsprachigen Gesellschaften für Sexualmedizin Standards in der Behandlung und Begutachtung von Transsexuellen formuliert und publiziert [1]. Diese Standards

\section{Abstract \\ $\nabla$}

Background: In 2011 the WPATH (World Professional Association for Transgender Health) published the $7^{\text {th }}$ version of their "Standards of Care" for diagnosis and treatment of transsexual people. In face of further recent peer-reviewed reports of experienced centres on surgical sex reassignment it should be examined whether or not genital sex reassignment in male-to-female transsexuals actually can be based on evidencebased guidelines or standards.

Results: The indication for surgery is widely standardised and evidence-based. Most critical steps of the operation are also founded on grade B recommendations. Most experienced authors rely on penoscrotal pedicled flaps for neovaginal lining. The topic of ideal reconstruction of the vulva, especially the clitoro-labial complex is still a field of debate. Due to the high frequency of further corrective surgeries which exceeds 50\% in most experienced centres, some authors prefer a primary 2-step procedure for genital reassignment.

Conclusions: The indication and principal operative steps in surgical genital reassignment in male-to-female patients rely on evidence-based recommendations. By respecting these recommendations subjective success rates of over $80 \%$ can be expected. 
wird [2]. Bezüglich der Empfehlungen zur operativen Genitalangleichung haben sich gegenüber der Version von 2001 keine wesentlichen Änderungen ergeben [3]. Unabhängig von den genannten Empfehlungen und Standards hat die International Society of Sexual Medicine (ISSM) im Jahre 2010 evidenzbasierte Empfehlungen nach einem 2-jährigen Konsensusprozess publiziert [4]. Ergänzend sind in den letzten Jahren mehrere Übersichtsarbeiten aus auf diesem operativen Gebiet spezialisierten Zentren erschienen, die insbesondere Empfehlungen zur operativen Technik präzisieren [5-10]. Der folgende Überblick soll eine aktuelle Standortbestimmung zum Stellenwert der operativen Genitalangleichung bei Mann-zu-Frau-Transsexuellen ermöglichen.

\section{Indikation zur Operation \\ $\nabla$}

Die Indikation zur operativen Genitalangleichung sollte von 2 unabhängigen auf diesem Gebiet erfahrenen Gutachtern gestellt werden [2]. Die Konstanz des transsexuellen Wunsches und die Lebbarkeit der angestrebten Geschlechtsrolle muss durch einen psychotherapeutisch begleiteten sogenannten „Alltagstest“ bewiesen werden. Eine ärztlich kontrollierte gegengeschlechtliche Hormontherapie sollte über mindestens 6 Monate präoperativ erfolgen. Eine Kostenübernahmeerklärung der gesetzlichen oder privaten Krankenversicherung sollte vorliegen und der Patient sollte das 18. Lebensjahr vollendet haben. Letzterer Punkt ist aktuell in Diskussion, da zunehmend Transsexualität bei Kindern und Jugendlichen frühzeitig diagnostiziert und behandelt wird [6]. Diesbezügliche deutschsprachige Leitlinien finden sich zurzeit im Status nascendi. Eine obere Altersgrenze für genitalangleichende Operationen besteht nicht [11], ebenso wenig gilt eine ruhende HIV-Erkrankung mit niedriger Viruslast als Kontraindikation [2]. Eine wesentliche Voraussetzung zur Operation ist die ausführliche präoperative Aufklärung durch den Operateur mit ausreichendem Abstand zur Operation und die realistische Einschätzung der Betroffenen bezogen auf die postoperativen Ergebnisse und möglichen Komplikationen [2]. Die Operation selbst sollte von einem auf diesem Gebiet erfahrenen Operateur durchgeführt werden, der grundsätzlich mehr als nur eine Operationsmethode beherrscht, um individuellen Besonderheiten gerecht werden zu können [2,7]. Hierbei ist es unrelevant, ob der Operateur Plastischer Chirurg, Gynäkologe, Urologe oder Allgemeinchirurg ist [2,3].

\section{Operationsmethoden \\ $\nabla$}

Grundsätzlich gestattet die evidenzbasierte Literaturanalyse einen Empfehlungsgrad B für die Ziele der Genitalangleichung [4] (siehe $\bullet$ Tab. 1).

Die Operation sollte die beidseitige hohe Orchiektomie beinhalten sowie die möglichst vollständige Resektion der Corpora cavernosae, die Schaffung einer ausreichend tiefen und weiten

\section{Tab. 1 Recommendation der ICSM [4].}

\section{Male-to-Female Genital Surgery Grade B}

Bilateral orchiectomy, amputation of the corpora cavernosa, creation of a neovaginal cavity that is lined by hairless skin, the formation of a sensate neoclitoris, and an aesthetic vulval appearance are the aims of genital surgery. The outcome may be achieved in one or two stages with satisfaction rates of $80 \%$ expected.
Neovagina, die Rekonstruktion einer sensiblen Neoklitoris, einer weiblichen Harnröhrenmündung und einer weiblich anmutenden Vulva [10]. Individuell kann natürlich auf Wunsch des Patienten (z.B. in höherem Lebensalter) auf Teile der Operation verzichtet werden (z.B. Anlage der Neovagina).

Viele Autoren bevorzugen eine penoskrotale Längsinzision mit Verlängerung ins Perineum in Form eines umgekehrten Y. Hierbei entsteht ein 3-eckförmiger perineal/skrotaler Lappen, der am Ende der Operation für die Rekonstruktion der hinteren Kommissur dient. Hoden und Samenstränge werden möglichst bis zum Leistenkanal verfolgt und nach Ligatur dort abgesetzt. Die Stümpfe werden im Leistenkanal versenkt. Der Penis wird zwischen Colle'scher und Buck'scher Faszie ausgehülst, danach wird entweder aus einem Teil der Glans oder der gesamten Glans die Neoklitoris präpariert. Im eigenen Vorgehen hat es sich bewährt, nur ein 3-eckförmgies dorsales Mittelstück der Glans zu verwenden und den Rest der Glans mit der Vorhaut wieder zu vernähen. So entsteht nach Umstülpen des penilen Hautschlauches eine Art „Neocollum“ am tiefsten Ende der Vagina und man gewinnt zusätzliche Länge der Vaginalauskeidung. Das dorsale Gefäßnervenbündel wird zusammen mit der Neoklitoris unter möglichst mikrochirurgischen Bedingungen von den Corpora nach Eröffnung der Buck'schen Faszie gelöst und die Corpora so tief als möglich auf den unteren Schambeinästen über Durchstechungsligaturen abgesetzt. Zuvor wurde die Urethra im unteren Penisschaftbereich durchtrennt und ebenfalls von den Corpora abpräpariert. Zur Schaffung der Neovagina ist der sauberste Zugang ein anatomisch exakt definierter Einstieg über das Zentrum tendineum [7]. Nach Durchtrennung desselben wird in extremer Steinschnittpositionierung der Musculus recto urethralis durchtrennt und auf der Dorsalseite der Prostata zwischen beiden Blättern der Denonvillier'schen Faszie eine Höhle geschaffen. Die so entstehende Höhle kann immer tief und weit gestaltet werden, ohne sich dem Risiko von Blutungen in dieser avaskulären Schicht auszusetzen. Die Möglichkeit einer sterilen Palpation über das Rektum erleichtert die Präparation und die Kontrolle auf Intaktheit des Rektums. Schließlich wird unter Einlage von Drainagen die penile Haut als Auskleidung der Neovaginalhöhle eingestülpt und von dorsal die hintere Kommissur durch Einnaht des perineal/skrotalen Lappens rekonstruiert. An typischer Stelle wird der Penishautschlauch eröffnet zum Durchzug der Neoklitoris nach torsions- und spannungsfreier Lagerung des dorsalen Gefäß-Nervenbündels. Nach Einnaht der Neoklitoris in die penile Haut erfolgt eine weitere Eröffnung der Penishaut zum Durchzug der gekürzten Urethra. Streifen des Corpus spongiosum mit Urethralschleimhaut können erhalten werden und zur Gestaltung des Vestibulums eingenäht werden, oder auch in die Neovaginalauskleidung integriert werden. Der Einschluss der Urethralschleimhaut in die Neovaginalauskleidung kann neben einer Umfangsvermehrung auch die Lubrifikation der Neovagina verbessern. Zusätzlich dürfte die Rate an postoperativen Meatusstenosen gesenkt werden. Eine saubere Blutstillung kann jedoch hierdurch erschwert sein. Um bei späterer sexueller Stimulation ein Anschwellen des Bulbus des Corpus spongiosum mit konsekutiver Einengung des Vaginaleingangs zu verhindern, empfiehlt sich eine partielle dorsale Resektion des Bulbus unter Beachtung der Harnröhrendurchgängigkeit [7]. Die noch vorhandene Skrotalhaut kann dann zur Rekonstruktion der großen und ggf. kleinen Schamlippen herangezogen werden.

Ob und wie weit schon während dieser Operation eine plastische Rekonstruktion der kleinen Schamlippen sowie andere Details 
Tab. 2 Komplikationen nach Mann-zu-Frau-Genitalangleichung (aus 5).

\begin{tabular}{|c|c|c|c|c|}
\hline & $\begin{array}{l}\text { Rossi et al. [5] } \\
n=332\end{array}$ & $\begin{array}{l}\text { Lawrence [14] } \\
n=232\end{array}$ & $\begin{array}{l}\text { Goddard [15] } \\
n=233\end{array}$ & $\begin{array}{l}\text { Baranyi [16] } \\
\text { Review }\end{array}$ \\
\hline \multicolumn{5}{|l|}{ Genitalregion } \\
\hline Striktur Vaginaleingang & $15 \%$ & & & $22 \%$ \\
\hline Entfernung Reste Schwellkörper & $15 \%$ & & & \\
\hline Vaginalstenosen & $12 \%$ & $8 \%$ & $6 \%$ & \\
\hline Verlust Vaginaltiefe & $8 \%$ & & $6 \%$ & $11 \%$ \\
\hline Dyspareunie & $2 \%$ & $9 \%$ & & $9 \%$ \\
\hline Part.Klitorisnekrose & $2 \%$ & $3 \%$ & & $10 \%$ \\
\hline Vaginalprolaps & $1 \%$ & & & \\
\hline \multicolumn{5}{|l|}{ Harntrakt } \\
\hline Harnröhrenstenose & $40 \%$ & $25 \%$ & $18 \%$ & \\
\hline Stenoserezidiv & $15 \%$ & $18 \%$ & $23 \%$ & \\
\hline Urethrafisteln & $4 \%$ & & & $11 \%$ \\
\hline \multicolumn{5}{|l|}{ Darmtrakt } \\
\hline rektale Verletzung & $3 \%$ & & & \\
\hline Wundheilungsstörung & $33 \%$ & & & \\
\hline anhaltende genitale Schmerzen & $3 \%$ & & & $9 \%$ \\
\hline
\end{tabular}

der Vulva angestrebt werden sollte, ist diskussionswürdig [4,5,710]. Die überwiegende Mehrzahl der Publikationen befürwortet die Verwendung des penilen Hautschlauchs für die Auskleidung der Vagina: penile Haut als gestielter Lappen ist sensibel in der Regel haarfrei und gut durchblutet. Probleme können entstehen bei Zustand nach radikaler Circumcision oder sehr kurzer Penislänge. In diesen Fällen kann der penile Hautschlauch mit freien Hauttransplantaten z.B. aus überschüssiger enthaarter Skrotalhaut verlängert werden. Einige Autoren, insbesondere in Thailand, befürworten primär die Verwendung freier Hauttransplantate für die Neovaginalauskleidung und nutzen die penile Haut zur plastischen Optimierung der kleinen Schamlippen und der Klitorisregion [7]. Naturgemäß haben freie Hauttransplantate jedoch eine höhere Schrumpfungstendenz als vaskularisierte Haut. Die primäre Verwendung von gestielten Darmsegmenten wird aufgrund zahlreicher gravierender Komplikationsmöglichkeiten und aufgrund der erhöhten Invasivität nicht empfohlen, kann jedoch in Zukunft an Bedeutung gewinnen, falls zunehmend jugendliche Patienten nach pubertätsstoppender und früher gegengeschlechtlicher Hormontherapie zur Operation anstehen. Bei diesen Patienten finden sich häufig hypotrophe juvenile Genitalien ohne ausreichend lange Penishaut [7].

\section{Komplikationen und Folgeeingriffe}

Genitalangleichende Operationen bei Mann-zu-Frau-Transsexualität sind komplexe und zeitaufwendige Beckenoperationen mit einem nicht unerheblichen Thrombose- und Embolierisiko. Literaturangaben zur Häufigkeit dieser Ereignisse fehlen. Insofern empfiehlt sich eine möglichst weitgehende Risikominimierung, z. B. durch Unterbrechung der Östrogentherapie und eine suffiziente medikamentöse und physikalische Thromboseprophylaxe, sowie durch eine sorgfältige Lagerung mit Verwendung von Beinschlingen oder Fußhaltern. Nachblutungen sind häufig und stammen in der Mehrzahl der Fälle aus den Resten des Corpus spongiosum der Harnröhre [7]. Hämatome im Schamlippenbereich prädisponieren zu Wundheilungsstörungen und bei Kompression zur Nekrose der gestielten Hautlappen [5,7]. Die Häufigkeit und Verteilung postoperativer Komplikationen ist aus einer kürzlich publizierten Zusammenstellung bedeutender operativer Zentren ersichtlich (siehe $\bullet$ Tab. 2) [5].
Im eigenen Patientengut von inzwischen 402 primär operierten Patientinnen spiegeln sich die in $\bullet$ Tab. 2 genannten Häufigkeiten wieder: 6\% der Patientinnen wurden wegen insuffizientem Vaginalvolumen revidiert und mit einer Spalthautauskleidung der vertieften Vagina versorgt, 28,4\% unterzogen sich einer Meatusplastik nach distaler Harnröhrenstenose und 0,8\% erhielten nach Ausbildung einer rektovaginalen Fistel eine temporäre Anus praeter Anlage [12].

Revisionsoperationen sind also häufig, sodass einzelne Autoren primär schon ein 2-zeitiges Vorgehen propagieren [5, 8]. Im eigenen Vorgehen wird zwar ein einzeitiges Vorgehen intendiert, jedoch werden bei mehr als 50\% der Patientinnen Korrekturoperationen erforderlich, zumeist wegen Meatusstenosen und Unzufriedenheit mit dem Erscheinungsbild der Vulva. Letzteres kann durch eine sogenannte Venushügelplastik mit Gestaltung der kleinen Schamlippen und der vorderen Kommissur korrigiert werden $[10,13]$.

Eine Optimierung des Erscheinungsbildes des äußeren Genitales war und ist Gegenstand zahlreicher Publikationen [17]. Bis heute existieren jedoch keine evidenzbasierten Empfehlungen oder Leitlinien hierzu $[2,7,10]$. Die Verwendung von peniler Haut für die Urethrarekonstruktion wird von einigen Autoren propagiert, komprommitiert jedoch die Verfügbarkeit für die Auskleidung der Vagina. Im Einzelfall ist hier immer ein Kompromiss erforderlich. Da es sowieso in der Mehrzahl der Fälle zu Korrekturoperationen kommt, bevorzugen wir in Analogie zu anderen führenden Zentren primär die Anlage einer verlässlichen Vaginaltiefe und -Auskleidung; die Feingestaltung des äußeren Erscheinungsbildes der Vulva kann dann in einer zweiten Sitzung in der Mehrzahl der Fälle zufriedenstellend gelöst werden $[5,7,10,13]$ (siehe $\bullet$ Abb. 1a, b).

Zusammenfassend kann aktuell bestätigt werden, dass die Sinnhaftigkeit genitalangleichender Operationen bei Mann-zu-Frau Transsexuellen durch die Evidenzlage der aktuellen Literatur bestätigt wird (Recommendation Level B ). Die prinzipiellen Schritte der Operation sind ebenfalls weitgehend standardisiert. Feinheiten zur Gestaltung des äußeren Genitale sowie zur 1- oder 2-zeitigkeit des operativen Vorgehens sind noch Gegenstand der Diskussion. Bei Anwendung der aktuellen operativen Techniken sind mehr als $80 \%$ der betroffenen Patienten mit dem erreichten Ergebnis subjektiv zufrieden. 

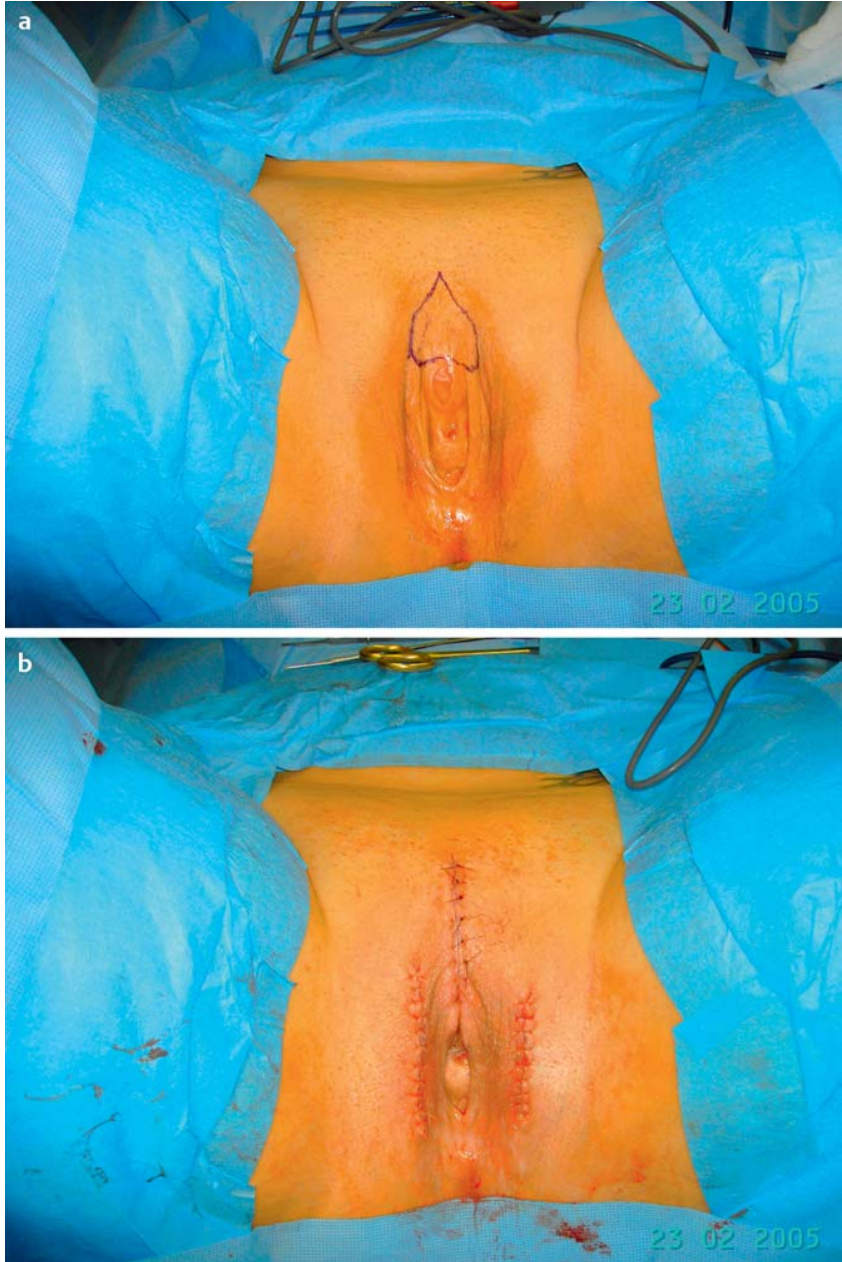

Abb. 1 a Zustand nach operativer Genitalangleichung vor 6 Monaten. Wunsch nach Venushügel und Schaffung eines Klitoris-Präputiums (Excisionsareal angezeichnet). b Zustand nach Venushügelplastik und Schaffung einer vorderen Kommissur.

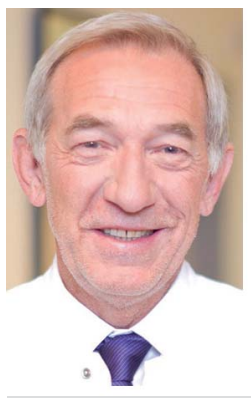

Prof. Dr. med. Michael Sohn

Geboren: 01.02.1955 in Vernon/Frankreich. Studium: 1974-1980: Studium der Humanmedizin in Bonn und Mendoza/Argent; November 1980: Erhalt der Approbation als Arzt.

Ausbildung: Januar 1981-März 1983: Assistenzarzt der Abteilung Urologie am Bundeswehr-Zentralkrankenhaus Hamburg (Flottenarzt Dr. med. Hartmann); April 1983-März 1984: Assistenzarzt der Abteilung Abdominal-, Gefäß- u. Unfallchirurgie am St. Johannes-Hospital in Bonn (Prof. Dr. med. Nedjabat); April 1984-Dez. 1984: Assistenzarzt der Abteilung Urologie des St. Elisabeth-Krankenhauses, Neuwied (Dr. med. Opelt); Januar 1985-März 1986: Assistenzarzt der Abteilung Urologie und Kinderurologie am St. Antonius-Hospital in Eschweiler (Prof. Dr. med. L. Steffens); April 1986-März 1987: Assistenzarzt, später stellvertr. Oberarzt an der Urologischen Univ.-Klinik des Insel-Spitals Bern (Prof. Dr. med. E. Zingg); Mai 1987-Sept. 1988: Oberarzt der Urol. Univ.-Klinik der RWTH Aachen (Prof. Dr. med. Lutzeyer, Kommiss. Direktor Dr. med. Deutz, Prof. Dr. med. Jak- se); Sept. 1988-Aug. 1995: Leitender Oberarzt der oben genannten Klinik; Seit Sept. 1995 Chefarzt der Urologischen Klinik am Markus-Krankenhaus gGmbH, Frankfurt a. M., Akademisches Lehrkrankenhaus der Johann-Wolfgang-GoetheUniversität, Frankfurt a. M.

Promotion: Dr. med.: 1983 in Bonn: mit der Note „MAGNA CUM LAUDE“; Thema der experimentellen Arbeit: Lebervenenverschlussdruckmessung vor und nach Oesophaguswandsklerosierung bei Oesophagusvarizen (Chirurgische Klinik der Univ.-Kliniken Bonn-Venusberg); Mai 1986: Anerkennung als Arzt für Urologie, Ärztekammer Nordrhein; 1993: Fellow of the European Board of Urology. Habilitation: am 29.01.1993: "Objektivierung organischer Erektionsstörungen und der Resultate mikrochirurgischer rekonstruktiver Verfahren“. a.p.l. Professur im Februar 1998: an der Medizinischen Fakultät der Univ. -Klinik Aachen; 2001-2003: Generalsekretär der ESSM; Seit 1988: Zahlreiche Studien zur Diagnostik und operativen und medikamentösen Therapie der erektilen Dysfunktion.

Interessenkonflikt: Nein

\section{Literatur}

1 Becker S, Bosinski HAG, Clemant $V$ et al. Standards der Behandlung und Geutachtung von Transsexuellen. Zschft f SexForsch 1997; 10: 147-156

2 Standards of Care for the Health of Transsexual, Transgender and Gender Nonconforming People. WPATH, $7^{\text {th }}$ version 2001; www.wpath. org

3 Monstrey S, Vercruysse H, De Cuypere G. Is Gender Reassignment Surgery Evidence Based? Recommendations for the Seventh Version of the WPATH Standards of care. Int J Transgenderism 2009; 11: 206-214

4 Ralph D, Gonzalez-Cadavid N, Sohn M et al. Trauma, Gender Reassignment and Penile Amputation. J Sex Med 2010; 7: 1657-1667

5 Rossi Neto R, Huitz F, Krege $S$ et al. Gender Reassignment Surgery - a 13 year review of surgical outcomes. JBJU 2012; 38: 97-107

6 Gooren LJ. Care of Transsexual Persons. N Engl J Med 2011; 364: 1251-1257

7 Selvaggi G, Bellringer J. Gender Reassignment Surgery: an overview. Nature Reviews Urology 2011; 8: 274-281

8 Imbimbo C, Verze P, Palmieri A et al. A Report from a Single Institutes 14-year-Experience in Treatment of Male-to-Female Transsexuals. J Sex Med 2009; 6: 2736-2745

9 Tuguet N, Goddard JC, Vicery RM et al. Current management of maleto-female gender identity disorder in the UK. Pmj. Bmj. Com 2012 www.postgradmedj.com

10 Sohn M, Bosinski HAG. Gender Identity Disorders: Diagnostic and Surgical Aspects. J Sex Med 2007; 4: 1193-1208

11 Rossi Neto R, van Dorp F, Schenck $M$ et al. Operative Geschlechtsangleichung im Alter. Urologe 2012; 51: 1414-1418

12 Sohn M. Complications in Transgender Genital Surgery. In: Austoni E (ed.). Atlas of Reconstructe Penile Surgery 2010; Pacini Editore, Pisa 455-458

13 Krege S, Bex A, Lümmen $G$ et al. Male-to-female transsexualis: a technique, results and long-term follow-up in 66 patients. BJU Internation 2001; 88: 396-402

14 Lawrence AA. Patient-Reported Complications and Functional Outcomes of Male-to-Female Sex-Reassignment Surgery. Arch Sex Behav 2006; 35: 717-727

15 Goddard JC, Vickery RM, Qureshi A et al. Feminizing genitoplasty in adult transsexuals: early and long-term surgical results. BJU Int 2007; 100: 607-613

16 Baranyi A, Piber D, Rothenhäusler HD. Mann-zu-Frau Transsexualismus: Ergebnisse geschlechtsangleichender Operationen in einer biopsychialen Perspektive. Wien Med Wochenschr 2009; 159: 548-557

17 Soli M, Brunocilla E, Bertuccini A et al. Male to Female Gender Reassignment: Modified Surgical Technique for Creating the Neoclitoris and Mons Veneris. J Sex Med 2008; 5: 210-216 